Rev. Biol. Trop., 48(2/3): 399-411, 2000

www.ucr.ac.cr www.ots.ac.cr www.ots.duke.edu

\title{
Distribución espacial de capturas de tiburón en el pacífico nicaragüiense y su relación con algunas variables oceanográficas
}

\author{
C.L.Brenes ${ }^{1}$, A. Hernández ${ }^{2} \& \mathrm{~J}$. Campos ${ }^{3}$ \\ 1 Laboratorio de Oceanografía y Manejo Costero Universidad Nacional, Heredia, Costa Rica, Apdo 86-3000 \\ Fax: (506) 26011 97, cbrenes@una.ac.cr \\ 2 ADPESCA, Managua, Nicaragua \\ 3 INRECOSMAR, San José, Costa Rica.
}

Recibido 26-IV-1999 Corregido 25-I-2000 Aceptado 4-II-2000.

\begin{abstract}
Between August 1995 and August 1997 long line fishing techniques and a bathythermograph were used to correlate some physical variables with the spatial distribution of four shark species in 26 fishing cruises off the Nicaraguan Pacific Coast. They were the thresher (Alopias vulpinus), blue (Prionace glaucea), gray (Carcharhinus falciformis) and hammer (Sphyra lewini). All species concentrated in the southeastern Nicaraguan Pacific, at the seasonal upwelling area of Papagayo Gulf. The range of sea surface temperatures in which the sharks were captured was 25$28^{\circ} \mathrm{C}$. We could clearly associate this physical parameter with shark availability. The vertical distribution of the captured sharks suggests that they occupy termocline levels above the $15^{\circ} \mathrm{C}$ isotherm. Although these species are oceanic, the blue shark was captured in ocean waters over the $1800 \mathrm{~m}$ isobar, while the grey and thresher sharks where close to the continental shelf. Body length in decreasing order are: thresher $\left(210-290 \mathrm{~cm}, \mathrm{SN}=2^{*} 1\right)$, blue $(60-240 \mathrm{~cm}, \mathrm{SN}=13)$ and gray $(80$ $200 \mathrm{~cm}, \mathrm{SN}=17$ ).
\end{abstract}

Key words: Fishery, sharks, thermal distribution, Nicaraguan pacific waters.

El Pacífico nicaragüense es una de las áreas menos estudiadas y dinámicamente más interesantes dentro de la complejidad que caracteriza a los patrones de circulación en el Pacífico Centroamericano. La aparición de afloramientos y remolinos fríos estacionales en sus aguas, interactuando con giros anticiclónicos cálidos localizados frente al Golfo de Fonseca son los responsables de un enriquecimiento casi permanente a través de todo el año de esta área del Pacífico Tropical (Brenes et al. 1995). La dinámica que en- vuelve la generación de estos remolinos cálidos y fríos desde la costa y su repercusión sobre la evolución del afloramiento en el Domo Térmico de Costa Rica no está aún totalmente determinada.

Los desplazamientos anuales en la dirección norte-sur de la Zona de Convergencia Intertropical (ZCIT) regulan la variabilidad estacional de las propiedades termohalinas de las masas de agua y de las corrientes superficiales en los trópicos. Esta zona de convergencia se mueve estacionalmente entre el 
ecuador en marzo-abril hasta $\sim 12^{\circ} \mathrm{N}$ en agosto y setiembre.

El extremo norte de la región frente al Golfo de Fonseca, es ocupado durante casi todo el año por aguas bastante cálidas $\left(\mathrm{T}>27.5^{\circ} \mathrm{C}\right)$. Estas aguas forman parte de una circulación anticiclónica que es característica de dicha área durante la mayor parte del año, la cual se ve debilitada eventualmente durante los últimos meses de cada año (Brenes 1998).

Durante los meses de verano (diciembremarzo) persiste en la zona un extenso núcleo de aguas frías con temperaturas inferiores a $26^{\circ} \mathrm{C}$, dentro de un patrón de circulación ciclónica. Los trabajos de Legeckis 1985 y Clarke 1988, permiten suponer que esta circulación ciclónica se extiende mucho más al sur hasta alcanzar la región frente al Golfo de Papagayo en Costa Rica.

En términos pesqueros, los resultados de varias campañas de prospección han mostrado que existen en esta zona biomasas considerables de importantes especies de valor comercial (PRADEPESCA 1996), sin embargo son muy pocos los trabajos que abarcan las posibles relaciones entre el comportamiento de algunas especies de interés comercial y factores abióticos.

En el presente trabajo se busca aportar información que permite de alguna manera, ir conociendo las posibles relaciones existentes entre algunos parámetros físicos del agua de mar, y el comportamiento de una especie de gran valor comercial en nuestros días, como es el tiburón.

\section{MATERIALES Y MÉTODOS}

Entre agosto de 1995 y agosto de 1997, bajo la cooperación científico-técnica y financiera del Gobierno de Japón y de la Agencia de Cooperación Internacional JICA, se desarrolló en Nicaragua el Proyecto "Pesca de Mediana Altura". El objetivo principal del citado Proyecto fue evaluar la pesquería de pelágicos en toda la Zona Económica Exclusiva (ZEE) del Pacífico de ese país. Con ese propósito se llevaron a cabo 26 cruceros a bordo de la embarcación MEDEPESCA II, con un total de 131 faenas de pesca (Anónimo 1998). Es uno de los pocos proyectos a nivel regional en el cual se obtuvieron mediciones simultáneas de parámetros físicos del agua de mar, lo que permitirá realizar algunos análisis en términos oceanográfico-pesqueros.

Las faenas de pesca se realizaron utilizando un palangre de deriva, y la temperatura del agua se determinó con un batitermógrafo Modelo ABT-1 de fabricación japonesa, con capacidad para registrar temperaturas y profundidades con una precisión de $0.05^{\circ} \mathrm{C}$ y 0.5 $\mathrm{m}$ respectivamente. El área donde se realizaron la mayoría de dichas faenas, se ubica dentro de la ZEE del Pacífico de Nicaragua, más allá de las 90 millas náuticas de distancia de la costa, entre los $200 \mathrm{~m}$ y los $3500 \mathrm{~m}$ de profundidad $\left(10^{\circ} 40^{\prime} \mathrm{N}\right.$ y $12^{\circ} 30^{\prime} \mathrm{N} ; 8^{\circ} 45-$ $88^{\circ} 45^{\prime} \mathrm{W}$ ) de longitud.

Para el Pacífico Tropical, la isoterma de $20^{\circ} \mathrm{C}$ se localiza en la mitad de la termoclina (Fiedler 1992). La profundidad de dicha isoterma se utilizó para representar la profundidad de la termoclina permanente (Donguy \& Meyers 1987).

\section{RESULTADOS}

La Fig. 1. muestra la posición de las zonas de pesca y la Fig. 2 presenta las zonas en las cuales se capturó tiburón. La Fig. 3 muestra la distribución espacial de los porcentajes de pesca (número de individuos por cada cien anzuelos) obtenidos para cada una de las especies de tiburón capturadas. En la Fig. 4 se muestran las distribuciones espaciales de la temperatura superficial y la profundidad de la termoclina, junto con los porcentajes de pesca para cada especie.

Como se puede apreciar en la Fig. 5, el mayor número de individuos capturados se 


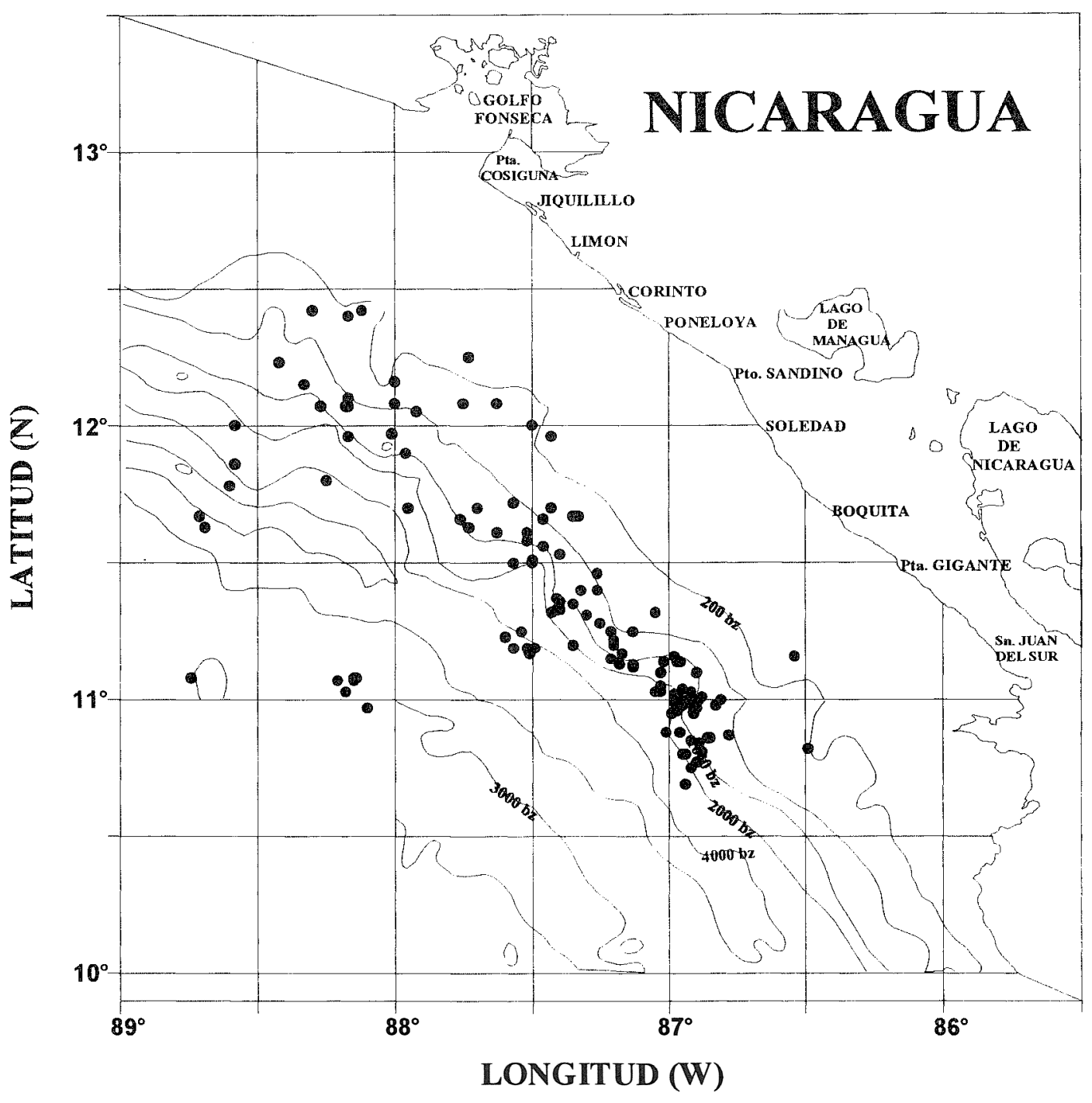

Fig. 1. Posición de las zonas de pesca.

Fig. 1. Position of the fishing zone. 


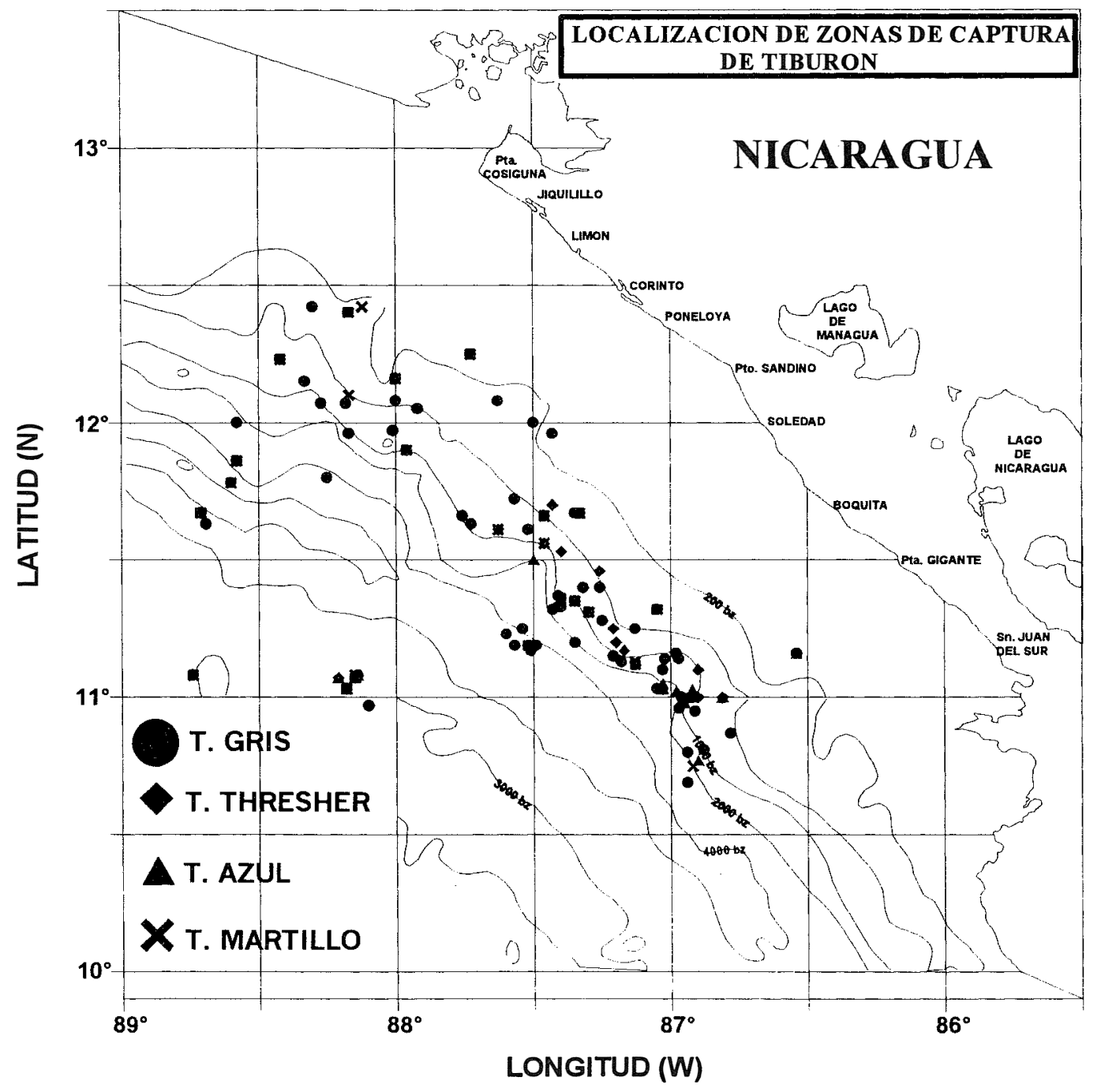

Fig. 2. Zonas de captura de tiburón.

Fig. 2. Shark capture zones. 

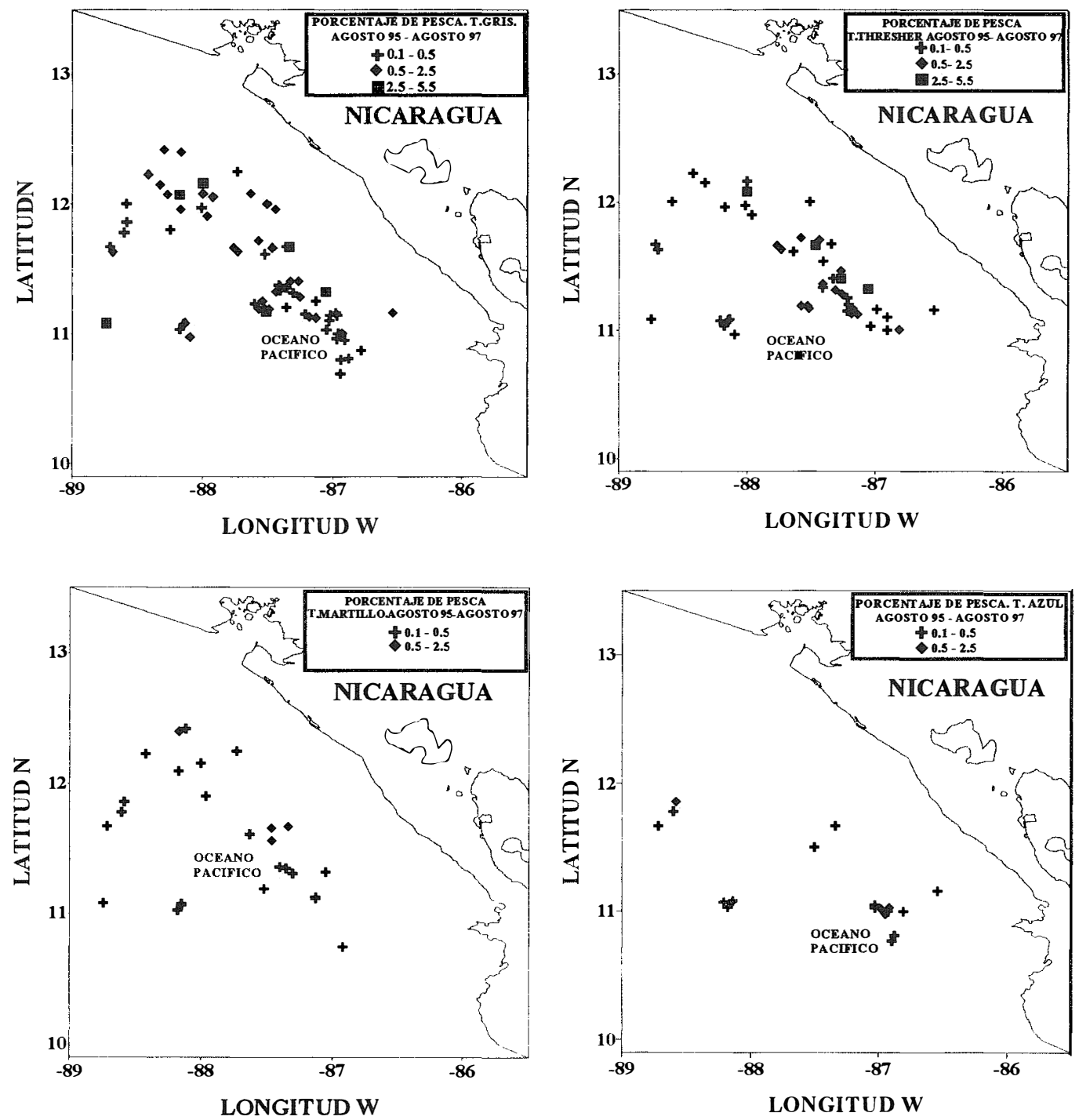

Fig. 3. Distribución espacial de los porcentajes de pesca por especie.

Fig. 3. Spatial distribution of fishing percentages by species. 

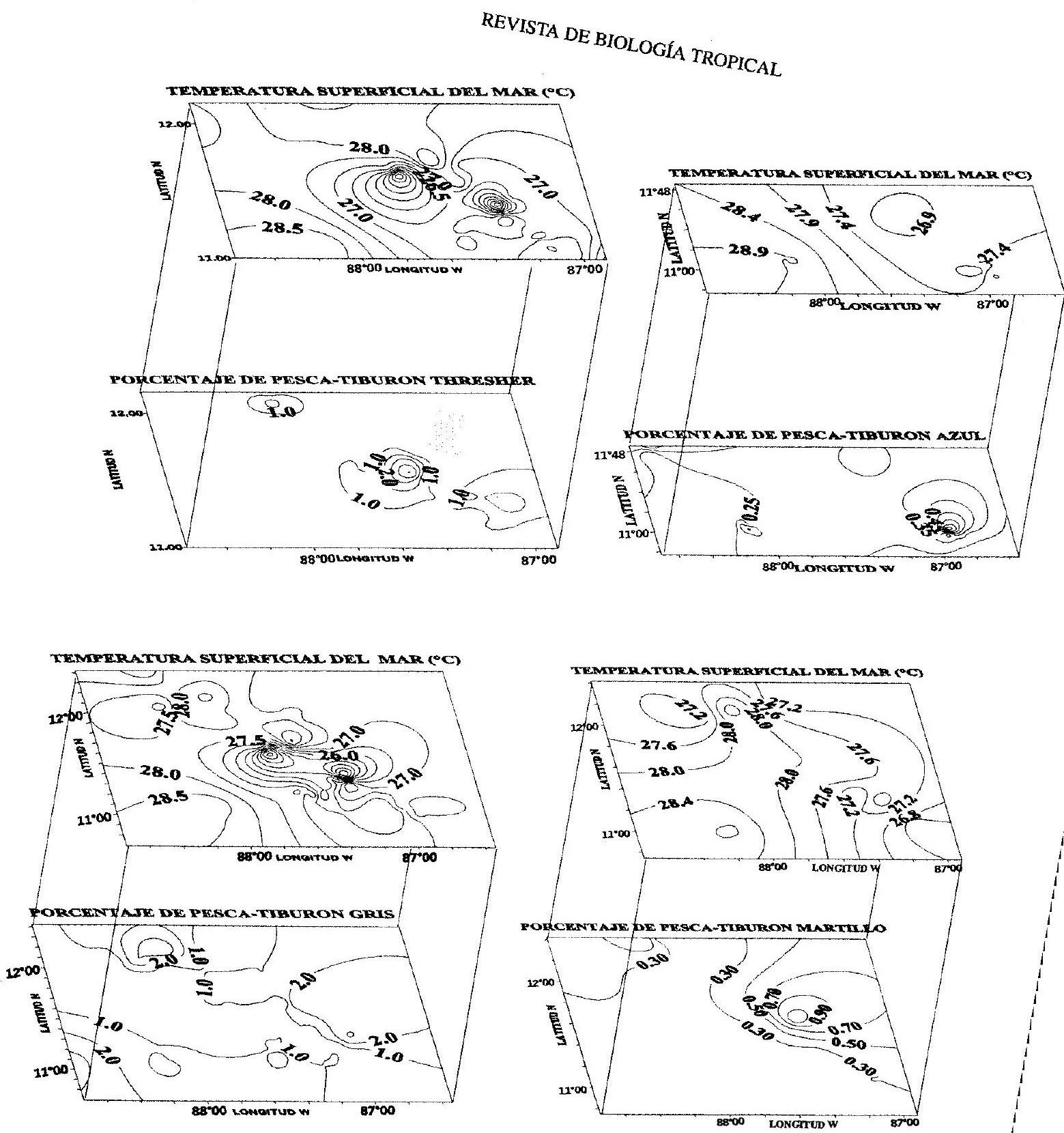

Fig. 4a.

capturadas en el Para superficia

Fig. 4a. Sea surface temperature $\left({ }^{\circ} \mathrm{C}\right)$, thermocline depth $(\mathrm{m})$ and fishing percentage
raguan Pacific.

$$
\text { (m) and fishing percentage for shark species captured in the Nica- }
$$




$$
\text { INTERNATIONAL JOURNAL OF TROPICAL BIOLOGY AND CONSERVATION }
$$
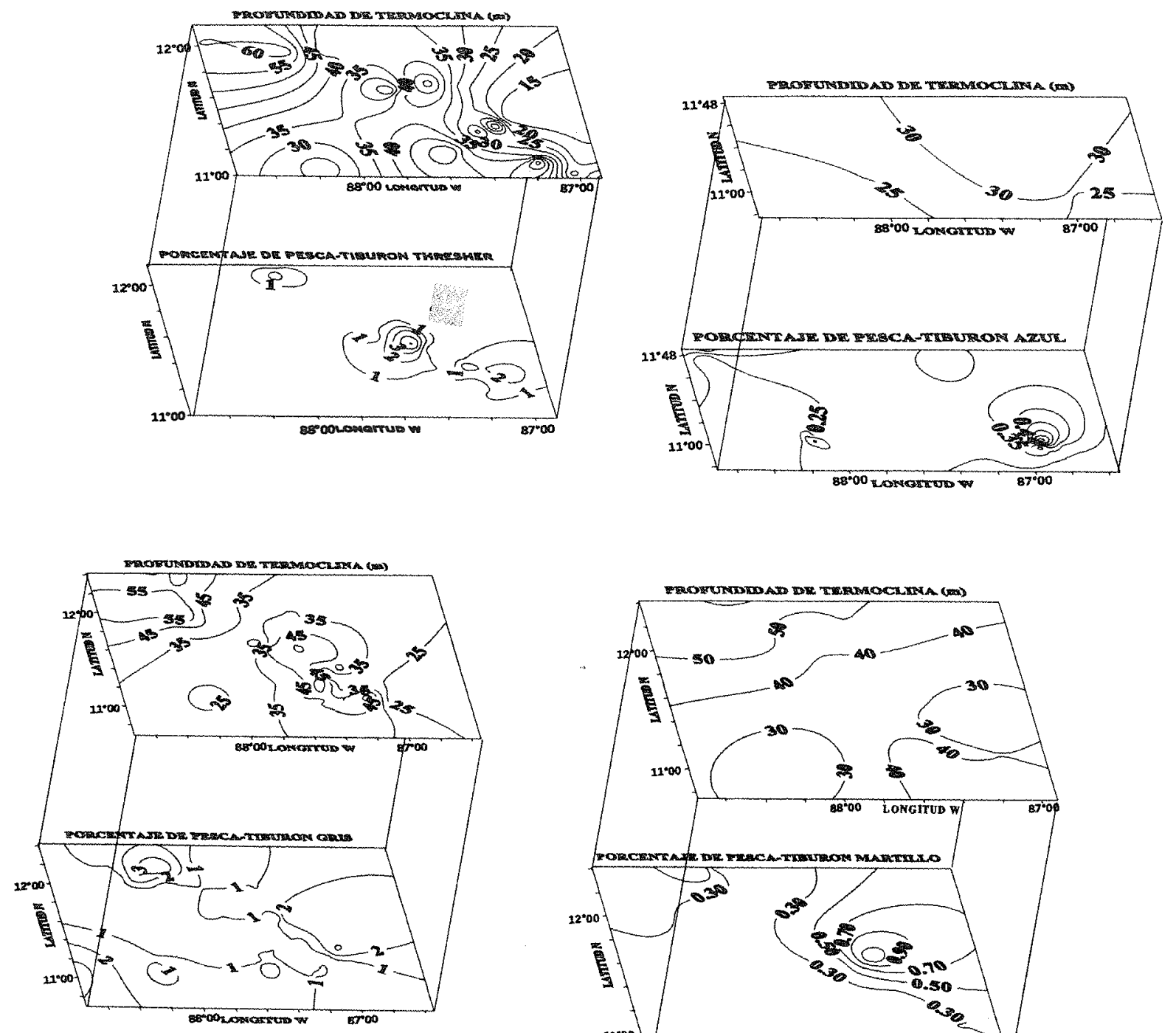

Fig. 4b. Temperatura

capturadas en el Pacífico de $N$ icicial, profundidad

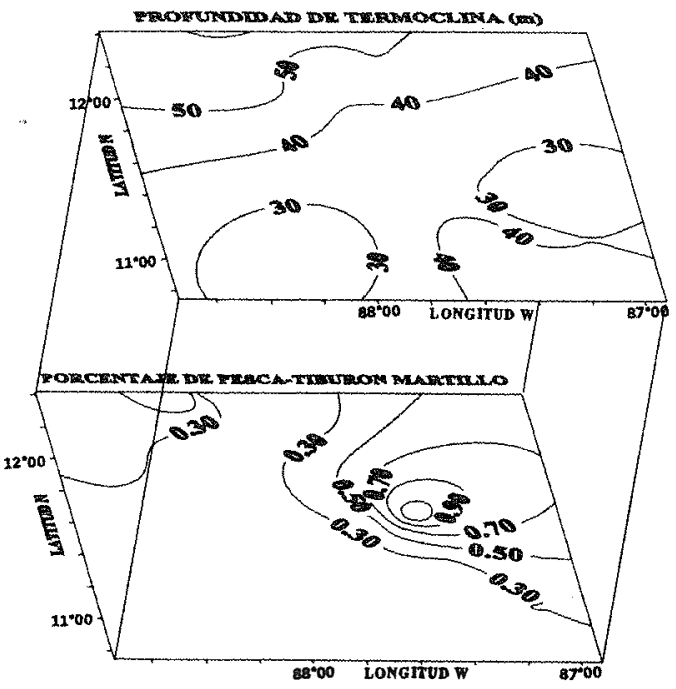
ig. 4 b. Sea surface temperature $\left({ }^{\circ} \mathrm{C}\right)$, thermocline depth $(\mathrm{m})$ and fishing percentage for shark
araguan Pacific. percentage for shark species captured in the $\mathrm{Ni}$. 
ubica sobre la isoterma de $20^{\circ} \mathrm{C}$, y en aquellas zonas en que la profundidad de dicha isoterma excede los $35 \mathrm{~m}$. El recurso se distribuye fundamentalmente sobre el estrato termoclino, y en muy pocas ocasiones se captura a temperaturas inferiores a los $15^{\circ} \mathrm{C}$. Cuando esto ocurre, las capturas son muy pequeñas (1-2 individuos por cada 100 anzuelos). Zonas con termoclinas muy someras $(<30 \mathrm{~m})$ muestran también capturas relativamente bajas en los niveles menos profundos.

La Fig. 6 presenta la distribución de tres especies de tiburón para los cuales se tienen datos de temperatura a los que fueron capturados. Los gráficos confirman lo que en forma general se aprecia en la Fig. 5, y es que el ám- bito de temperatura de captura efectivamente se ubica entre los 15 y $30^{\circ} \mathrm{C}$.

Al comparar el tiburón gris y el tiburón azul, éstos muestran un patrón inverso entre sí. Para la primera especie el aumento de individuos acompaña al aumento térmico, mientras que para el tiburón azul, el número de individuos disminuye conforme la temperatura del agua aumenta. Esto se explica por ser el tiburón azul una especie que se distribuye a mayores profundidades. El tiburón que a falta de nombre castellano llamaremos thresher muestra un comportamiento en el cual la tendencia general es un aumento del número de individuos con la temperatura, sobre todo a partir de $\operatorname{los} 20^{\circ} \mathrm{C}$.

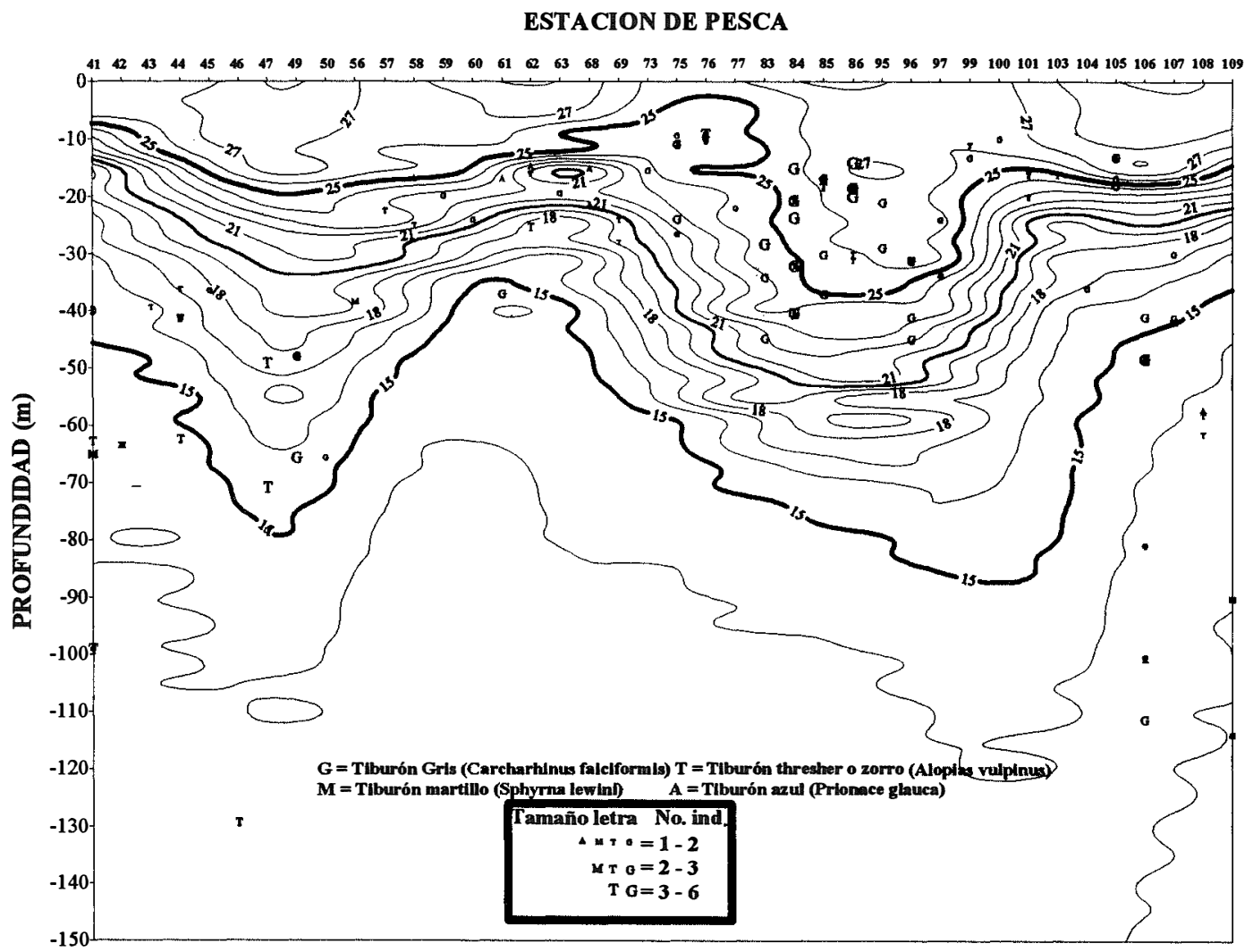

Fig. 5. Distribución vertical del número de individuos capturados por especie en función de la profundidad (m) y de la temperatura $\left({ }^{\circ} \mathrm{C}\right)$. Las isotermas de $15^{\circ} \mathrm{C}$ y $25^{\circ} \mathrm{C}$ definen el estrato termoclino.

Fig. 5. Vertical distribution of the number of sharks captured as a function of depth $(\mathrm{m})$ and temperature $\left({ }^{\circ} \mathrm{C}\right)$. The termocline is defined by the $15^{\circ} \mathrm{C}$ and $25^{\circ} \mathrm{C}$ isotherms. 

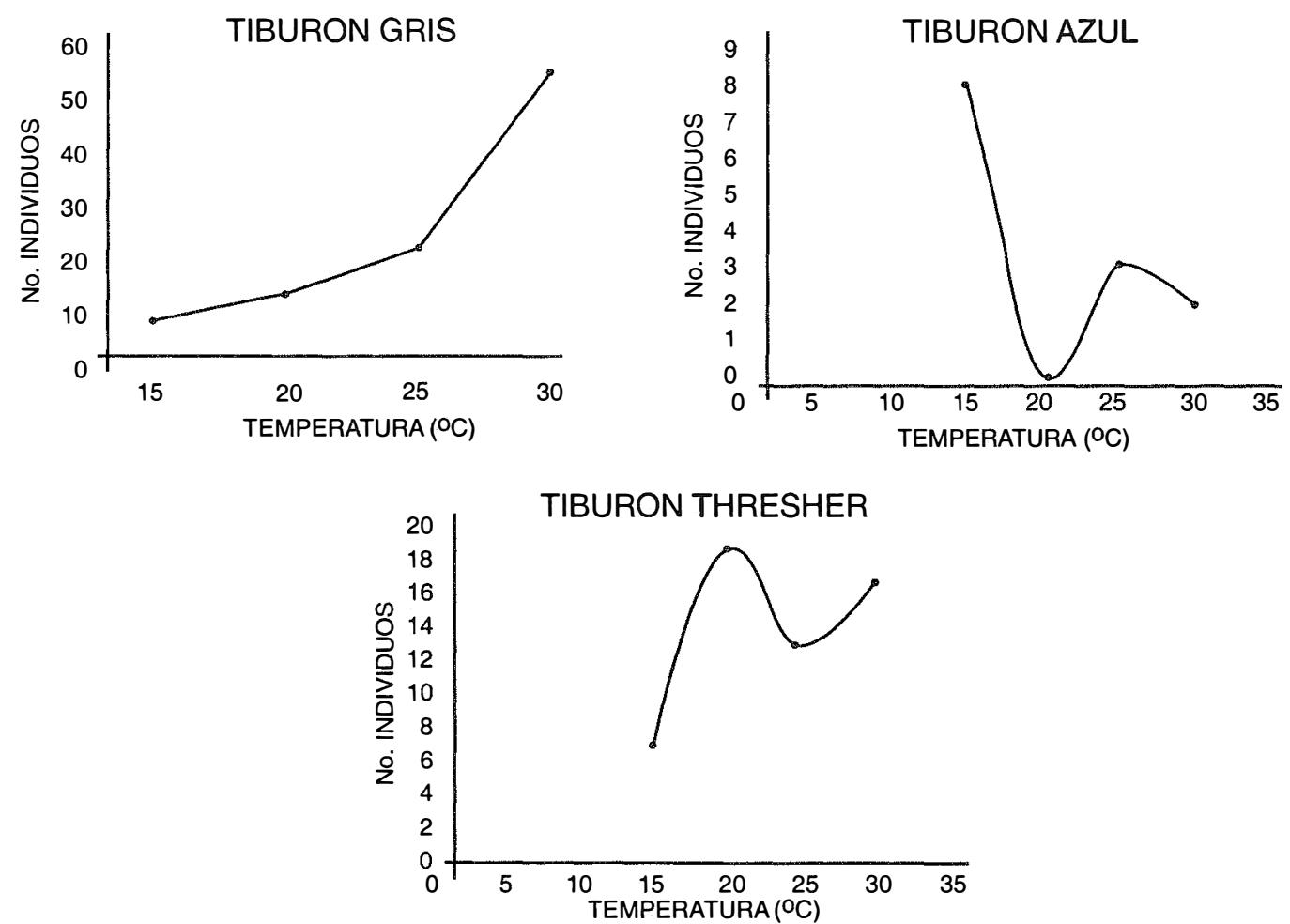

Fig. 6. Número de individuos por especie en función de la temperatura del agua $\left({ }^{\circ} \mathrm{C}\right)$.

Fig. 6. Sharks captured as a function of temperature $\left({ }^{\circ} \mathrm{C}\right)$ by species.

La Fig. 7 muestra la distribución vertical del número de individuos por tallas en relación con la profundidad. No se presenta la totalidad de los individuos capturados, debido a que no siempre se tenía el dato que correlacionara la talla con la profundidad. La mayoría de los tiburones thresher (A) tuvieron una talla entre los 250 y $260 \mathrm{~cm}$ de longitud, y fueron capturados entre los 40 y los $90 \mathrm{~m}$ de profundidad.
Las longitudes de los tiburones azul y gris (B) y (C) nunca superaron los $240 \mathrm{~cm}$. La profundidad de captura para estas dos especies rara vez superó los $100 \mathrm{~m}$ de profundidad. El tiburón azul se encontró sobre los 30 $\mathrm{m}$ y el gris sobre los $60 \mathrm{~m}$ de profundidad. El tiburón azul mostró las longitudes más pequeñas (alrededor de los $80 \mathrm{~cm}$ ). El tiburón gris no exhibe ningún patrón definido en su distribución vertical por tallas. 

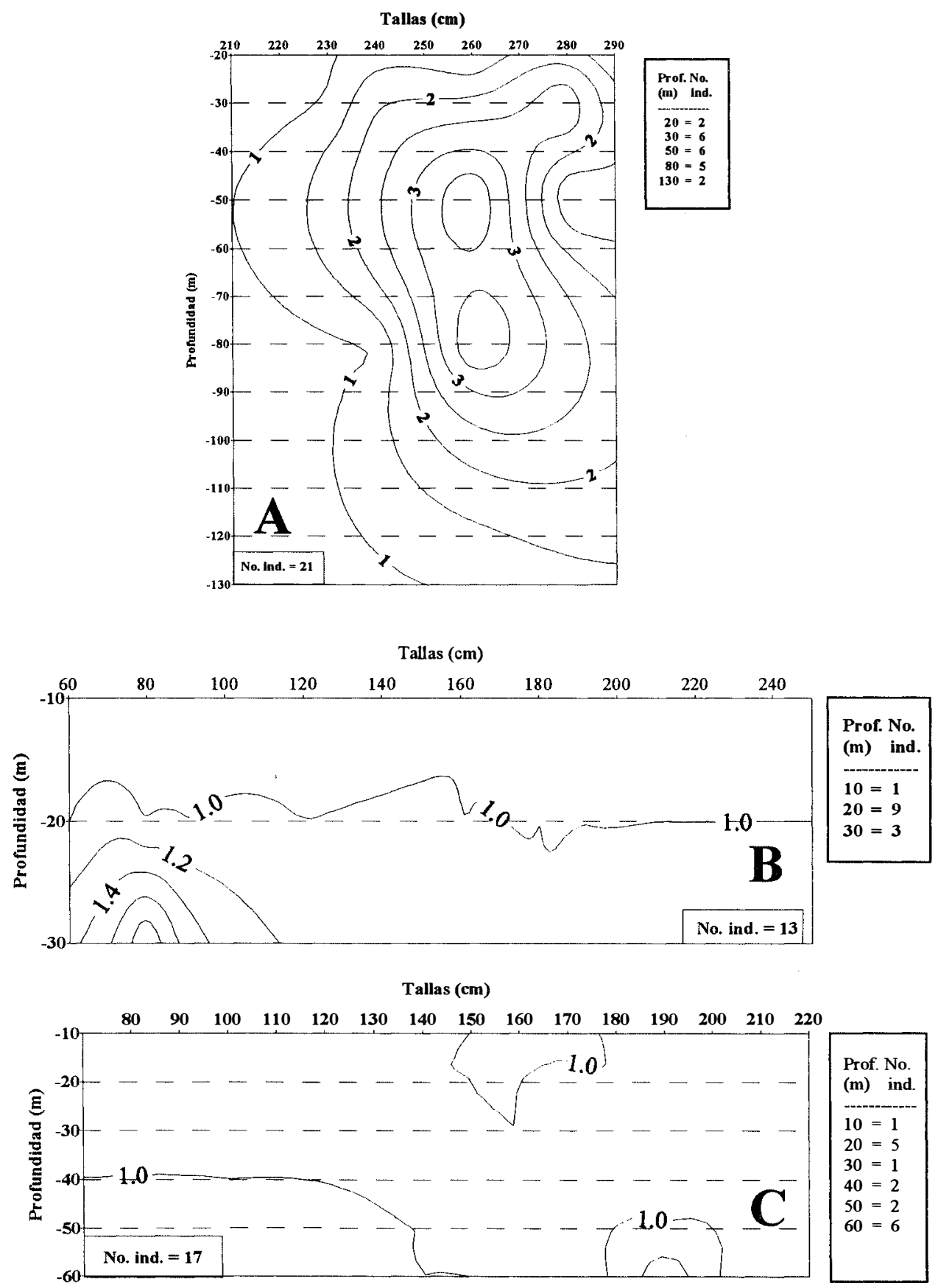

Fig. 7. Distribución vertical de las capturas de tiburón thresher (A), azul (B) y gris (C) por ámbito de tallas.

Fig. 7. Vertical distribution of sharks captured by size: thresher (A), blue (B) and grey (C). 


\section{DISCUSIÓN}

La mayor concentración en las capturas de tiburón thresher, azul y gris se dio en el extremo sureste del Pacífico Nicaragüense, en la región donde las aguas superficiales son relativamente bajas durante todo el año, comparadas con el resto del área (Fig. 2). Esta es una zona en la cual la presencia de frentes térmicos asociados a la interacción de remolinos fríos y cálidos es muy frecuente (Brenes 1998).

Un análisis de las distribuciones espaciales de las capturas en función de la profundidad local muestra resultados interesantes. En primer lugar, el tiburón azul, Prionace glauca, fue capturado en áreas donde la profundidad excedió los $1800 \mathrm{~m}$. Este tipo de tiburón es uno de los tiburones oceánicos-epipelágicos más abundantes en los océanos del mundo, y trabajos previos (Hazin et al. 1990, Crow et al. 1996) han mostrado que las capturas de esta especie han sido mayores en áreas donde la profundidad excede los $1000 \mathrm{~m}$. Estos mismos resultados habían sido reportados desde hace cuatro décadas por Strassburg (1958).

La distribución espacial de las capturas de tiburón gris, carcharhinus falciformis, es más amplia. Presenta sin embargo, un porcentaje significativo ( 20\%) en áreas menos profundas, coincidiendo con los resultados reportados por Hazin et al en 1990, en los cuales esta especie muestra una distribución claramente relacionada con el borde de la plataforma continental.

El tiburón thresher es mayormente oceánico, sin embargo, los resultados obtenidos por Straussbur en 1958 y por Porras (1996), revelaron que esta especie de tiburón tiende a acercarse a la plataforma continental o aglomerarse alrededor de islas oceánicas.

Los mayores porcentajes de pesca de tiburón thresher (2.5-5.5) se localizan en la región sureste del pacífico nicaragüense. En el caso del tiburón azul estos porcentajes no superaron los 2.5 , un valor bajo si se compara con los resultados de la especie anterior, observándose una mayor concentración al este de los $87^{\circ} \mathrm{W}$ sobre los $11^{\circ} \mathrm{N}$ de latitud.
Para el tiburón gris, la distribución espacial de los porcentajes de pesca es más homogénea que las anteriores (Fig. 3). No obstante, se observa una pequeña concentración en el extremo sur del Pacífico de Nicaragua, entre los $86^{\circ} 40-87^{\circ} 30 \mathrm{~W}$ y los $10^{\circ} 50-11^{\circ} 20 \mathrm{~N}$, y otra región centrada en $88^{\circ} 00 \mathrm{~W}-12^{\circ} 00 \mathrm{~N}$ en la zona más al norte. Finalmente, para el tiburón martillo, los resultados no muestran una zona clara de aglutinamiento. Se da una distribución muy homogénea a lo largo de todo el Pacífico Nicaragüense, sin ninguna tendencia claramente definida en el mapa.

El mayor porcentaje de pesca del tiburón thresher se obtuvo entre los $25^{\circ} \mathrm{C}$ y los $27^{\circ} \mathrm{C}$. La profundidad de la termoclina (isoterma de $20^{\circ} \mathrm{C}$ ) se localizó entre los 30 y $45 \mathrm{~m}$ en la zona de mejor captura. Este resultado nos indica que la termoclina no debe estar muy cerca de la superficie (lo cual implicaría aguas superficiales frías) para obtener mejores capturas. Cuando la termoclina se localiza en los niveles más profundos ( $>50 \mathrm{~m})$, los porcentajes de pesca disminuyen drásticamente en más del $75 \%$, asociados a la presencia de un estrato superficial muy cálido $\left(\mathrm{T}>28^{\circ} \mathrm{C}\right)$, lo cual explica la distribución de capturas observada en la Fig. 3.

Los mayores porcentajes de pesca del tiburón azul, se obtuvieron cuando las temperaturas superficiales estuvieron entre $\operatorname{los} 27^{\circ} \mathrm{C}$ y los $27.4^{\circ} \mathrm{C}$, y la profundidad de la termoclina se localizó entre los 25 y los $30 \mathrm{~m}$, un poco más somera que en el caso anterior. Porcentajes de pesca superiores a 2, para el tiburón gris, estuvieron entre los $26^{\circ} \mathrm{C}$ y $\operatorname{los} 28^{\circ} \mathrm{C}$, con una profundidad de la termoclina entre los 25 y $50 \mathrm{~m}$. Para ambos parámetros los ámbitos fueron mayores que en las dos especies anteriores.

Para el tiburón martillo, las temperaturas superficiales que mayor porcentaje de pesca produjeron oscilaron entre los $26.8^{\circ} \mathrm{C}$ y los $27.8^{\circ} \mathrm{C}$, con la termoclina localizada entre los 30 y $40 \mathrm{~m}$ de profundidad.

El Cuadro 1 presenta las temperaturas superficiales y las profundidades de la termoclina, para cada una de las especies estudiadas. 


\section{CUADRO 1}

Temperaturas superficiales y profundidad de termoclina asociadas con la captura de las diferentes especies de tiburón.

Especie de tiburón

Thresher

Azul

Gris

Martillo
Temperatura superficial $\left({ }^{\circ} \mathrm{C}\right)$

$25-27$

27-27.4

26-28

26.8-27.8
Profundidad de termoclina (m)

$30-45$
$25-30$
$25-50$
$30-40$

En el Atlántico Brasileño, la captura de tiburón azul fue en su mayoría en los niveles termoclinos y esporádicamente bajo la termoclina. Debe tenerse en cuenta que en esta parte del Océano Atlántico, la isoterma de $28^{\circ} \mathrm{C}$ define el tope de la termoclina y $\operatorname{los} 13.5^{\circ} \mathrm{C}$ su base (Hazin et al. 1993). Estos resultados concuerdan con los obtenidos en el presente análisis.

Adicionalmente, Crow et al. (1996) analizaron estadísticas de capturas de tiburón obtenidas por Programas Pesqueros de longline en Hawaii. La profundidad a la que se capturó tiburón gris, Carcharhinus falciformis, raramente excedió los $40 \mathrm{~m}$, mientras que el tiburón martillo, Sphyrna lewini, se capturó hasta los 143 m. Según Compagno (1984), la captura de esta última especie ha sido reportada desde la superficie hasta los $275 \mathrm{~m}$ de profundidad. Los resultados obtenidos en el presente trabajo, muestran también para estas especies, niveles de concordancia bastante buenos con los resultados reportados por dichos autores.

\section{RESUMEN}

Entre agosto de 1995 y agosto de 1997, se llevaron a cabo 26 campañas de pesca en el Pacífico de Nicaragua. Utilizando el palangre como arte de pesca y un batitermógrafo, fue posible relacionar algunas variables físicas con la distribución espacial y vertical de las diferentes especies de tiburón capturadas: thresher (Alopias vulpinus), azul (Prionace glauca), gris (Carcharhinus falciformis) y martillo (Sphyrna lewini). La distribución espacial de todas las especies mostró una significativa aglomeración en el extremo sureste del Pacifico nicaragüense, justo en el área de influencia del afloramiento estacional del Golfo de Papagayo. El ámbito máximo de temperaturas superficiales en las que se efectuaron las capturas se ubicó entre los $25^{\circ} \mathrm{C}$ y los $28^{\circ} \mathrm{C}$. Se logró determinar una clara dependencia entre este parámetro físico y la presencia del recurso. La distribución vertical de las capturas evidenció que estas especies se localizan principalmente en los niveles termoclinos, sobre la isoterma de $15^{\circ} \mathrm{C}$. En cuanto a la dependencia de las capturas con la profundidad local, el tiburón azul se capturó en áreas fundamentalmente oceánicas, sobre la isóbata de $1800 \mathrm{~m}$, mientras que el tiburón gris y thresher a pesar de ser también oceánicos, mostraron una tendencia a acercarse a la plataforma continental. Finalmente, en cuanto a las tallas, los registros mostraron que los especímenes con mayor talla pertenecieron al tiburón thresher, con longitudes entre los $200 \mathrm{y}$ los $300 \mathrm{~cm}$. Le siguió en orden descendente el tiburón gris y el tiburón azul.

\section{AGRADECIMIENTOS}

Agradecemos al grupo de funcionarios y trabajadores de MEDEPESCA que participaron en el Proyecto Pesca de Mediana Altura, y a Akiya Seko, asesor de la Agencia de Cooperación Japonesa (JICA), sin los cuales hubiera sido imposible contar con tan valiosa información. A la Universidad Nacional de Costa Rica por el apoyo al Proyecto de Investigación "Servicio Regional de Información Oceanográfica”.

\section{REFERENCIAS}

Anónimo. 1996. Campaña de Pesca Comercial Simulada. Informe. Panamá: 115 .

Anónimo. 1998. La pesquería de peces pelágicos en el Océano Pacífico de Nicaragua. Proyecto Pesca de Mediana Altura (PMA). Nicaragua: 124 p. 
Brenes, C.L., B. Kwiecinski, L. D`Croz \& J. Chaves. 1995. Características oceanográficas de la plataforma pacífica de América Central y aguas oceánicas adyacentes: Cruceros 25 set-18 oct 1993, 25 feb-18 marzo 1994. Informe de campañas. PRADEPESCA, Panamá, 77 p.

Brenes, C.L., A. Hernández \& A.Gutiérrez. 1998. Sea surface thermohaline variations along the Nicaraguan Pacific coastal waters. Rev. Tópicos Meteorológicos y Oceanográficos 5: 17-27.

Campagno, L.J.V. 1984. Sharks in the world: An annotated and illustrated bibliography of species known to date. FAO Species Catalogue No 4. parts 1 and 2. FAO, Roma.

Clarke, A. 1988. Inertial Wind Path and the Sea Surface Temperature Pattern Near the Gulf of Tehuantepec and Gulf of Papagayo. J. Geophys. Res. 93(C12): 15491-155501

Crow, G, C. Lowe \& B. Wetherbee. 1996. Shark Records from Longline Fishing Programs in Hawaii with Comments on the pacific Ocean Distributions. Pac. Sci. 50: 382-392.

Donguy, J.R \& G.Meyers. 1987. Observed and modelled topography of the $20^{\circ} \mathrm{C}$ isotherm in the tropical Pacific. Oceanol. Acta 10: 41-48.
Fiedler, P.C. 1992. Seasonal Climatologies and Variability of Eastern Tropical Pacific Surface Waters. NOAA Thecnical Report NMFS 109.

Hazin, H.V, A. Couto, K. Kihara, K. Otsuka \& M. Ishino. 1990. Distribution and abundance of pelagic sharks in the South-Western Equatorial Atlantic. J. Tokio Univ. Fish. 77: 51-64.

Hazin, H.V, C. Boeckman, E. Leal, R. Lessa, K. Kihara \& K. Otsuka. 1993. Distribution and relative abundance of the blue shark, Prionace glauca, in the South-Western Equatorial Atlantic. J.fishery Bull. 92: 474-480.

Legeckis, R. 1985. Upwelling off the Gulfs of Panamá and Papagayo in the Tropical Pacific during March 1985. J. Geophys. Res. 93 (C12): 15485-15489.

Porras, O. 1996. Campañas de pesca comercial simulada realizadas con palangre en la zona económica exclusiva del litoral pacífico de Panamá, El Salvador y Guatemala: Informe. PRADEPESCA, Panamá, 91 p.

Strassburg, D.W. 1958. Distribution, abundance and habits of pelagic sharks in the central Pacific Ocean. U.S. Fish. Wildl..Serv., Fish. Bull. 58: 335-361. 